Research Article

\title{
The Complex Dynamics of Sharing Platform Competition Game
}

\author{
Jianli Xiao $\mathbb{D}^{1,2}$ \\ ${ }^{1}$ Management School, Fudan University, Shanghai 200433, China \\ ${ }^{2}$ School of Business Administration, Nanchang Institute of Technology, Nanchang 330099, China \\ Correspondence should be addressed to Jianli Xiao; 17110690001@fudan.edu.cn
}

Received 30 October 2021; Accepted 6 December 2021; Published 22 December 2021

Academic Editor: Juan L. G. Guirao

Copyright (c) 2021 Jianli Xiao. This is an open access article distributed under the Creative Commons Attribution License, which permits unrestricted use, distribution, and reproduction in any medium, provided the original work is properly cited.

\begin{abstract}
With the rapid development of Internet technologies and online sharing platforms, sharing economy has become a major trend in economy. The entry of sharing economy leads to profound impacts on incumbent industry. We build a dynamic sharing platform competition model with which agents are bounded rational, and consumer side is heterogeneous. Then, we present the fixed points and the stability conditions of the bifurcation of the dynamic model. We simulate the adjustment speed of sharing platform, sharing platform price, and costs of traditional firm effects on system stability, and we present stable area, bifurcation diagram, the largest Lyapunov exponent, and strange attractor of different parameters, and we give a feedback control method at last. Our main results are as follows: (1) when adjustment speed of sharing platform increases, the system becomes bifurcation, and finally, the system goes into a chaotic state; when the system is stable, price of traditional firm and fee decision of sharing platform are constant. (2) When price of sharing platform increases, sharing platform is more stable while traditional firm is more vulnerable. Suppose the system is in the stable state; when sharing platform price increases, traditional firm price increases, while sharing platform fees decreases. (3) When traditional firm cost is small, the system would be more stable. When the system is stable, with traditional firm cost increasing, traditional firm price increases quicker than sharing platform consumer fee, while sharing platform seller fee decreases. (4) Feedback control can alleviate the chaotic state of system. With feedback control parameter increases, the system becomes more stable.
\end{abstract}

\section{Introduction}

Sharing economy has become a growing trend in world [1]. With sharing platforms, consumers have access to products/ services which they do not own [2]. Sharing platforms reduce transaction costs, such as searching costs and replication costs [3]. Sharing platforms compete with traditional firm fiercely, such as the entry of ride-sharing platforms leads to the significant loss of traditional industry in profit [4]. The entry of bike-sharing platforms leads to the intense competition in China in 2020 and the decrease of $68 \%$ in manufacturers' profits [1]. However, although sharing platform improves the resource efficiency, there are lots of shortages in sharing platform, for example, the business stealing effect on traditional firms [5] and traffic jam caused by car-sharing. Therefore, the mechanism behind the chaos, which is activated by sharing economy, should be concerned.
Sharing economy is characterized by its accessible economy through platforms [6]. Its two-sided market structure becomes the core characteristic factors. Sharing platform is a two-sided market; one group in one side of platforms would increase its utility when the other side group number increases. The fee decision-making of the sharing platform is bounded rational [7], for its lack of information. Sharing platform competition is studied by many researchers. Pei et al. [8] investigate the sharing economy $\mathrm{B} 2 \mathrm{C}$ and $\mathrm{C} 2 \mathrm{C}$ platform price strategy. Tian and Jiang [9] investigate the different effects of sharing platforms on retailers and find that, according to their marginal costs, sharing would benefit more on retailers. The sharing platforms such as Uber is a two-sided platform, and they have the intragroup externality between different groups, so they have to charge two-sided problems, such as multihoming problems, and conquer and divide strategy. Cachon et al. [10] compare surge of different pricing policies in ride 
sharing. Seamans and Zhu [11] study the dynamic price in platform and show the entry of platforms leads to more difference between newspapers.

Two-sided markets' theory is first described by Rochet and Tirole [12]; then, a lot of literature is investigated. Armstrong [13] analyzes the two-sided market pricing strategy and finds that pricing is determined by externality, charging method, and multihoming. Bardey et al. [14] show that quality affects the price structure in the two-sided market. Belleflamme and Toulemonde [15] study the intragroup negative externality model in two-sided market externality. Ambrus and Argenziano [16] study an asymmetric network in two-sided markets.

Sharing economy creates many features for government [17], for it is innovative [18, 19]. We focus on the sharing platform that have no power on pricing, and it gets maximum profit by taking the fees on two sides in sharing platform. Sharing platforms charge for their services on different sides of the sharing platform. The price of the service in the sharing platform is determined by exogenous factors, such as the secondary books' exchange. He and Shen [20] investigate e-hailing service and traditional taxi in a spatial equilibrium model. Ye et al. [21] show that sharing platform competes with the traditional firm.

Sharing platforms have to make decisions on how to charge consumers and developers. However, the current studies neglect that the sharing platforms are two-sided markets. Sharing platforms have to attract more consumers or firms to increase its externality and profits. Sharing platforms always have access to more consumers than tradition industry.

Another relevant stream of literature is about the chaos dynamics. Andaluz et al. [22] compare price competition and quantity competition in evolution game and find that quantity competition is more stable. Du et al. [23] analyze the duopoly game with heterogeneous players and find that, with the output limiter, the system becomes more stable. Fanti and Gori [24] analyze the duopoly game with heterogeneous product, higher degree of product differentiation may lead to a chaotic state, and more fiercer competitions lead market to be less stable. Dubiel-Teleszynski [25] analyzes the diseconomy of scale in competition and finds that small scale destabilizes the market equilibrium. Pyragas [26] describes the new development of feedback control.

However, to the best of our knowledge, there have been no attempts to consider the sharing platform competition as a two-sided platform. We combine the sharing platform competition and the chaos theory. Zou and He [27] study technology sharing through technology innovation platform. Our paper is different from their study for our model is about sharing economy; our model considers sharing economy as a two-sided market, and the sharing economy market structure is shown in Figure 1; our model has new features.

This paper investigates a nonlinear discrete-time game model with sharing platform and traditional firm competition. We assume two players are bounded rationality expectations. Through simulation of the complex dynamical

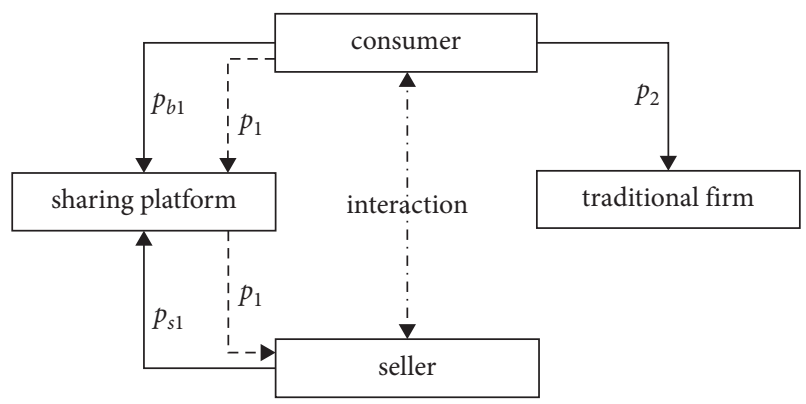

FIgURE 1: Market structure.

behaviors, we present a feedback chaos control and find that it can alleviate the chaos state.

The remainder of this paper is organized as follows. In Section 2, we present a competition model consisting of a sharing platform and a traditional firm. In Section 3, we discuss the Nash equilibrium points and stability region. In Section 4, numerical simulations are carried out, and we show the major features of this game. We give the feedback control for the chaotic state. Finally, a conclusion is given.

\section{Model}

We illustrate the sharing platform competition model in Figure 1. Consumer and seller interact with each other through sharing platform, and sharing platform charges fees on the consumer and the seller. Traditional firm compete with the sharing platform in the consumer side.

There are new features in our model. First, different from Pei et al. [8] and Ye et al. [21], we see the sharing platform as a two-sided market. Consumer utility depends on the quantity of sellers. Second, we assume the consumer facing a given product price, which is common in sharing economy. The sharing platform has no power on price. Third, we take sharing platform's decision on fee as a whole, and the sharing platform competes with traditional firm only in the consumer side.

We assume that sharing platform and traditional firm compete in a Hoteling model, assuming that consumers distribute, in a unit interval $[0,1]$, sharing platform and tradition firm positioning in two ends. We use 1 to stand for sharing platform and use 2 to stand for the traditional firm. The consumer who in location $x$ buys product incurs transportation cost $t, t>0$. For simplicity, we assume $t$ equals to 1 . As in [28], we assume sharing price $p_{1}$ in sharing platform is exogenous. Sharing platform charges consumers' fee $p_{b 1}$ and sellers' fee $p_{s 1}$ to maximize its profit. $p_{2}$ is the price of traditional product. $v$ is the instinct value that the consumer consumes the product. The sharing platform and firm compete to attract the same group of consumers. The consumer who joins sharing platform utility function is

$$
u_{b 1}=v+\alpha q_{s 1}-p_{b 1}-p_{1}-x,
$$

where $\alpha$ is the network externality parameter; in the sharing platform, consumer utility depends on sellers quantity who joins sharing platform, price, and transaction costs. 

is

The consumer who joins traditional firm utility function

$$
u_{2}=v-p_{2}-(1-x),
$$

where $u_{2}$ is traditional firm utility and $p_{2}$ is the price of traditional firm. In traditional firm, consumer utility depends on price of traditional firm and transaction costs.

In the seller side, we assume the seller's utility function that joins sharing platform is

$$
u_{s 1}=q_{b 1} p_{1}-p_{s 1}-c_{1}
$$

where $c_{1}$ is sellers' cost.

For simplicity, we assume that seller's cost distribute in a unit line $[0,1]$. When $u_{s 1}>0$, the seller will attend the sharing platform. So, the seller quantity is $q_{s 1}=\operatorname{Pr}\left(q_{b 1} p_{1}-p_{s 1}-c_{1}>0\right)$; then, we can calculate the seller's number is

$$
q_{s 1}=q_{b 1} p_{1}-p_{s 1}
$$
that

From equations ( 1 ) and (2), let $u_{b 1}=u_{2}$; we can conclude

$$
\begin{aligned}
q_{b 1} & =\frac{1}{2}+\frac{\alpha q_{s 1}+p_{2}-p_{1}-p_{b 1}}{2}, \\
q_{2} & =\frac{1}{2}-\frac{\alpha q_{s 1}+p_{2}-p_{1}-p_{b 1}}{2} .
\end{aligned}
$$

The sharing platform makes the decision of the fees on the consumer side and the developer side as a whole. The seller's fee decision depends on the consumer side's fee decision. From equations (4) and (5), we can obtain

$$
\left\{\begin{array}{l}
q_{b 1}=\frac{1+p_{2}-p_{1}-\alpha p_{s 1}-p_{b 1}}{2-\alpha p_{1}}, \\
q_{s 1}=\frac{p_{1}\left(1+p_{2}-p_{1}-p_{b 1}\right)-2 p_{s 1}}{2-\alpha p_{1}} .
\end{array}\right.
$$

The sharing platform's profit function is

$$
\pi_{1}=p_{b 1} q_{b 1}+p_{s 1} q_{s 1}
$$

where $q_{b 1}$ is the quantity of the consumer who joins the sharing platform.

The traditional firm's profit function is

$$
\pi_{2}=\left(p_{2}-c_{2}\right) q_{2}
$$

where $q_{2}$ is the quantity of traditional firm consumer and $c_{2}$ is the traditional firm's marginal costs.

We insert equation (7) in profit function (8), and the first-order condition for the maximization of the sharing platform's profit is

$$
\frac{\partial \pi_{1}}{\partial p_{s 1}}=\frac{p_{1}\left(1+p_{2}-p_{1}\right)-\left(p_{1}+\alpha\right) p_{b 1}-4 p_{s 1}}{2-\alpha p_{1}}=0
$$
is

Then, the sharing platform seller's fee reaction function

$$
p_{s 1}=\frac{p_{1}\left(1+p_{2}-p_{1}\right)-\left(p_{1}+\alpha\right) p_{b 1}}{4} .
$$

Combine equations (7) and (11), and then, substitute into equation (8); the first-order condition equation that maximizes sharing platform profit is

$$
\frac{\partial \pi_{1}}{\partial p_{b 1}}=\frac{-\left(8-\left(\alpha+p_{1}\right)^{2}\right) p_{b 1}+\left(4-\alpha p_{1}-p_{1}^{2}\right) p_{2}+p_{1}^{3}-(1-\alpha) p_{1}^{2}-(4+\alpha) p_{1}+4}{4\left(2-\alpha p_{1}\right)}
$$

Combine equations (6) and (11), and then, substitute into equation (9); the first-order condition equation that maximizes traditional firm profit is

$$
\frac{\partial \pi_{2}}{\partial p_{2}}=\frac{\left(4-\alpha p_{1}-\alpha^{2}\right) p_{b 1}-\left(8-2 \alpha p_{1}\right) p_{2}-\alpha p_{1}^{2}-\left(3 \alpha+\alpha c_{2}-4\right) p_{1}-4 c_{2}+4}{4\left(2-\alpha p_{1}\right)}
$$

As Choi et al. [28], we assume the sharing platform and traditional firm are bounded rational and obtain the following system:

$$
\left\{\begin{array}{l}
p_{b 1}(t+1)=p_{b 1}(t)+\lambda_{1} p_{b 1}(t) \frac{\partial \pi_{1}}{\partial p_{b 1}} \\
p_{2}(t+1)=p_{2}(t)+\lambda_{2} p_{2}(t) \frac{\partial \pi_{2}}{\partial p_{2}}
\end{array}\right.
$$

where $\lambda_{1}$ is the sharing platform's adjustment speed of consumer fee, $\lambda_{1}>0$, and $\lambda_{2}$ is the adjustment speed of traditional firm price, $\lambda_{2}>0$. In the following we will analyze the equilibrium of the dynamic model.

\section{Dynamic Model Analysis}

By setting $p_{b 1}(t+1)=p_{b 1}(t)$ and $p_{2}(t+1)=p_{2}(t)$, we can obtain 4 fixed points: 
$E 1(0,0)$

$$
\begin{aligned}
& E 2\left(0, \frac{4-\left(3 \alpha p_{1}-4 c_{2}-4 p_{1}\right)-\alpha p_{1}\left(p_{1}+c_{2}\right)}{8-2 \alpha p_{1}}\right) \\
& E 3\left(\frac{p_{1}^{3}-(1-\alpha) p_{1}^{2}-(4+\alpha) p_{1}+4}{8-\left(\alpha+p_{1}\right)^{2}}, 0\right) \\
& E 4\left(\frac{\left(4-\alpha p_{1}-p_{1}^{2}\right)\left(12+\left(4-\alpha p_{1}\right) c_{2}+\alpha p_{1}^{2}-5 \alpha p_{1}-4 p_{1}\right)}{48+\alpha p_{1}\left(\alpha+p_{1}\right)^{2}-4\left(\alpha^{2}+6 \alpha p_{1}-p_{1}^{2}\right)}, \frac{\left(4-\alpha p_{1}\right)\left(8-\left(\alpha+p_{1}\right)^{2}\right) c_{2}+4\left(2-\alpha p_{1}\right)\left(6+2 p_{1}-\left(\alpha+p_{1}\right)^{2}\right)}{48+\alpha p_{1}\left(\alpha+p_{1}\right)^{2}-4\left(\alpha^{2}+6 \alpha p_{1}-p_{1}^{2}\right)}\right)
\end{aligned}
$$

System (14) Jacobian matrix is

$$
J=\left(\begin{array}{cc}
1+\lambda_{1}\left(\frac{\partial \pi_{1}}{\partial p_{b 1}}+p_{b 1} \frac{\partial^{2} \pi_{1}}{\partial p_{b 1}^{2}}\right) & \lambda_{1} p_{b 1} \frac{\partial^{2} \pi_{1}}{\partial p_{b 1} \partial p_{2}} \\
\lambda_{2} p_{2} \frac{\partial^{2} \pi_{2}}{\partial p_{b 1} \partial p_{2}} & 1+\lambda_{2}\left(\frac{\partial \pi_{2}}{\partial p_{2}}+p_{2} \frac{\partial^{2} \pi_{2}}{\partial p_{2}^{2}}\right)
\end{array}\right) .
$$

Let $\operatorname{Tr}$ be the trace of Jacobian matrix and Det be the determent of Jacobian matrix. Then, we can obtain

$$
\begin{aligned}
\operatorname{Tr} & =2+\lambda_{1}\left(\frac{\partial \pi_{1}}{\partial p_{b 1}}+p_{b 1} \frac{\partial^{2} \pi_{1}}{\partial p_{b 1}^{2}}\right)+\lambda_{2}\left(\frac{\partial \pi_{2}}{\partial p_{2}}+p_{2} \frac{\partial^{2} \pi_{2}}{\partial p_{2}^{2}}\right) \\
\operatorname{Det} & =\left(1+\lambda_{1}\left(\frac{\partial \pi_{1}}{\partial p_{b 1}}+p_{b 1} \frac{\partial^{2} \pi_{1}}{\partial p_{b 1}^{2}}\right)\right)\left(1+\lambda_{2}\left(\frac{\partial \pi_{2}}{\partial p_{2}}+p_{2} \frac{\partial^{2} \pi_{2}}{\partial p_{2}^{2}}\right)\right)-\lambda_{1} \lambda_{2} p_{b 1} p_{2} \frac{\partial^{2} \pi_{1}}{\partial p_{b 1} \partial p_{2}} \frac{\partial^{2} \pi_{2}}{\partial p_{b 1} \partial p_{2}}
\end{aligned}
$$

The determinant equation is

$$
x^{2}-\operatorname{Tr} x+\operatorname{Det}=0 .
$$

The $E 1$ point Jacobian matrix is

$$
\left(\begin{array}{cc}
1+\lambda_{1} \frac{p_{1}^{3}-(1-\alpha) p_{1}^{2}-(4+\alpha) p_{1}+4}{4\left(2-\alpha p_{1}\right)} & 0 \\
0 & 1+\lambda_{2} \frac{4-\left(3 \alpha p_{1}-4 c_{2}-4 p_{1}\right)-\alpha p_{1}\left(p_{1}+c_{2}\right)}{4\left(2-\alpha p_{1}\right)}
\end{array}\right)
$$

The characteristic roots are $1+\lambda_{1}\left(\left(p_{1}^{3}-(1-\alpha) p_{1}^{2}-\right.\right.$ $\left.\left.(4+\alpha) p_{1}+4\right) / 4\left(2-\alpha p_{1}\right)\right)$ and $1+\lambda_{2}\left(\left(4-\left(3 \alpha p_{1}-4 c_{2}-\right.\right.\right.$ $\left.\left.\left.4 p_{1}\right)-\alpha p_{1}\left(p_{1}+c_{2}\right)\right) / 4\left(2-\alpha p_{1}\right)\right)$. For $\left(\left(p_{1}^{3}-(1-\alpha) p_{1}^{2}-\right.\right.$ $\left.\left.(4+\alpha) p_{1}+4\right) / 4\left(2-\alpha p_{1}\right)\right)>0$ and $\left(\left(4-\left(3 \alpha p-4 c_{2}-4 p\right)\right.\right.$ $\left.\left.-\alpha p\left(p+c_{2}\right)\right) / 4(2-\alpha p)\right)>0$, so $1+\lambda_{1}\left(\left(p_{1}^{3}-(1-\alpha) p_{1}^{2}-\right.\right.$ $\left.\left.(4+\alpha) p_{1}+4\right) / 4\left(2-\alpha p_{1}\right)\right)>1$ and $1+\lambda_{2}\left(\left(4-\left(3 \alpha p_{1}-4 c_{2}\right.\right.\right.$ $\left.\left.\left.-4 p_{1}\right)-\alpha p_{1}\left(p_{1}+c_{2}\right)\right) / 4\left(2-\alpha p_{1}\right)\right)>1 ; E 1$ is repelling node [29].

$E 2\left(0,\left(4-\left(3 \alpha p_{1}-4 c_{2}-4 p_{1}\right)-\alpha p_{1}\left(p_{1}+c_{2}\right)\right) /(8-2 \alpha\right.$ $\left.\left.p_{1}\right)\right)$ point Jacobian matrix is 


$$
\left(\begin{array}{cc}
1+\lambda_{1}\left(\frac{\left.\left(4-\alpha p_{1}-p_{1}^{2}\right) p_{2}^{a}+p_{1}^{3}-(1-\alpha) p_{1}^{2}-(4+\alpha) p_{1}+4\right)}{4(2-\alpha p)}\right) & 0 \\
\lambda_{2} p_{2}^{a} \frac{4-\alpha p_{1}-p_{1}^{2}}{4\left(2-\alpha p_{1}\right)} & 1+\lambda_{2} p_{2}^{a} \frac{4-\alpha p_{1}-p_{1}^{2}}{4\left(2-\alpha p_{1}\right)}
\end{array}\right)
$$

where $\quad p_{2}^{a}=\left(4-\left(3 \alpha p_{1}-4 c_{2}-4 p_{1}\right)-\alpha p_{1}\left(p_{1}+c_{2}\right)\right) /(8-$ $\left.2 \alpha p_{1}\right)$.

For $\quad\left(\left(\left(4-\alpha p_{1}-p_{1}^{2}\right) p_{2}^{a}+p_{1}^{3}-(1-\alpha) p_{1}^{2}-(4+\alpha) p_{1}+\right.\right.$ 4) $/ 4(2-\alpha p))>0$, so $1+\lambda_{1}\left(\left(\left(4-\alpha p_{1}-p_{1}^{2}\right) p_{2}^{a}+p_{1}^{3}-(1-\right.\right.$ $\left.\left.\alpha) p_{1}^{2}-(4+\alpha) p_{1}+4\right) / 4(2-\alpha p)\right)>1$.
For $\left(\left(4-\alpha p_{1}-p_{1}^{2}\right) / 4\left(2-\alpha p_{1}\right)\right)<0, \quad p_{2}^{a}>0$, so $1+\lambda_{2}$ $p_{2}^{a}\left(\left(4-\alpha p_{1}-p_{1}^{2}\right) / 4\left(2-\alpha p_{1}\right)\right)<1$.

So, $E 2$ is a saddle point [29].

E3 $\left(\left(\left(p_{1}^{3}-(1-\alpha) p_{1}^{2}-(4+\alpha) p_{1}+4\right) / 8-\left(\alpha+p_{1}\right)^{2}\right), 0\right)$ point Jacobian matrix is

$$
\left(\begin{array}{cc}
1+\lambda_{1} p_{b 1}^{b} \frac{4-\alpha p_{1}-\alpha^{2}}{4\left(2-\alpha p_{1}\right)} & \lambda_{1} p_{b 1}^{b} \frac{4-\alpha p_{1}-\alpha^{2}}{4\left(2-\alpha p_{1}\right)} \\
0 & 1+\lambda_{2} \frac{\left(4-\alpha p_{1}-\alpha^{2}\right) p_{b 1}^{b}-\alpha p_{1}^{2}-\left(3 \alpha+\alpha c_{2}-4 t\right) p_{1}-4 c_{2}+4}{4\left(2-\alpha p_{1}\right)}
\end{array}\right)
$$

where $\quad p_{b 1}^{b}=\left(\left(p_{1}^{3}-(1-\alpha) p_{1}^{2}-(4+\alpha) p_{1}+4\right) / 8-(\alpha+\right.$ $\left.p_{1}\right)^{2}$.

For $p_{b 1}^{b}>0, \quad\left(\left(4-\alpha p_{1}-\alpha^{2}\right) / 4\left(2-\alpha p_{1}\right)\right)<0$, so $1+\lambda_{1}$ $p_{b 1}^{b}\left(\left(4-\alpha p_{1}-\alpha^{2}\right) / 4\left(2-\alpha p_{1}\right)\right)<1$.

When $p_{b}^{b}>\left(\left(\alpha p_{1}^{2}+\left(3 \alpha+\alpha c_{2}-4 t\right) p_{1}+4 t c_{2}-4\right) / 4-\alpha\right.$ $\left.p_{1}-\alpha^{2}\right)$, then $\left(\left(\left(4-\alpha p_{1}-\alpha^{2}\right) p_{b}^{b}-\alpha p_{1}^{2}-\left(3 \alpha+\alpha c_{2}-4 t\right)\right.\right.$ $\left.\left.p_{1}-4 t c_{2}+4\right) / 4\left(2-\alpha p_{1}\right)\right)>0$, so $1+\lambda_{2}\left(\left(\left(4-\alpha p_{1}-\alpha^{2}\right)\right.\right.$ $\left.\left.p_{b}^{b}-\alpha p_{1}^{2}-\left(3 \alpha+\alpha c_{2}-4 t\right) p_{1}-4 c_{2}+4\right) / 4\left(2-\alpha p_{1}\right)\right)>1 ; E 3$ is the saddle point.

When $p_{b}^{b}<\left(\left(\alpha p_{1}^{2}+\left(3 \alpha+\alpha c_{2}-4 t\right) p_{1}+4 t c_{2}-4\right) /(4-\right.$ $\left.\left.\alpha p_{1}-\alpha^{2}\right)\right)$, then $\left(\left(\left(4-\alpha p_{1}-\alpha^{2}\right) p_{b}^{b}-\alpha p_{1}^{2}-\left(3 \alpha+\alpha c_{2}-4 t\right)\right.\right.$ $\left.\left.p_{1}-4 t c_{2}+4\right) / 4\left(2-\alpha p_{1}\right)\right)<0$, so $+\lambda_{2}\left(\left(\left(4-\alpha p_{1}-\alpha^{2}\right) p_{b}^{b}-\alpha\right.\right.$ $\left.\left.p_{1}^{2}-\left(3 \alpha+\alpha c_{2}-4 t\right) p_{1}-4 c_{2}+4\right) / 4\left(2-\alpha p_{1}\right)\right)<1 ; E 3$ is an attracting point, and $E 3$ is stable.
$E 4\left(\left(\left(4-\alpha p_{1}-p_{1}^{2}\right)\left(12+\left(4-\alpha p_{1}\right) c_{2}+\alpha p_{1}^{2}-5 \alpha p_{1}-\right.\right.\right.$ $\left.\left.4 p_{1}\right) / 48+\alpha p_{1}\left(\alpha+p_{1}\right)^{2}-4\left(\alpha^{2}+6 \alpha p_{1}-p_{1}^{2}\right)\right),\left(\left(4-\alpha p_{1}\right)(8\right.$ $\left.-\left(\alpha+p_{1}\right)^{2}\right) c_{2}+4\left(2-\alpha p_{1}\right)\left(6+2 p_{1}-\left(\alpha+p_{1}\right)^{2}\right) /\left(48+\alpha p_{1}\right.$ $\left.\left.\left.\left(\alpha+p_{1}\right)^{2}-4\left(\alpha^{2}+6 \alpha p_{1}-p_{1}^{2}\right)\right)\right)\right)$ point Jacobian matrix is

$$
\left(\begin{array}{cc}
1-\lambda_{1} p_{b 1}^{c} \frac{8-\left(\alpha+p_{1}\right)^{2}}{4\left(2-\alpha p_{1}\right)} & \lambda_{1} p_{b 1} \frac{4-\alpha p_{1}-p_{1}^{2}}{4\left(2-\alpha p_{1}\right)} \\
\lambda_{2} p_{2}^{c} \frac{4-\alpha p_{1}-\alpha^{2}}{4\left(2-\alpha p_{1}\right)} & 1-\lambda_{2} p_{2}^{c} \frac{8-2 \alpha p_{1}}{4\left(2-\alpha p_{1}\right)}
\end{array}\right)
$$

where $p_{b 1}^{c}=\left(\left(4-\alpha p_{1}-p_{1}^{2}\right)\left(12+\left(4-\alpha p_{1}\right) c_{2}+\alpha p_{1}^{2}-5 \alpha\right.\right.$ $\left.\left.p_{1}-4 p_{1}\right) /\left(48+\alpha p_{1}\left(\alpha+p_{1}\right)^{2}-4\left(\alpha^{2}+6 \alpha p_{1}-p_{1}^{2}\right)\right)\right)$,

$$
p_{2}^{c}=\frac{\left(4-\alpha p_{1}\right)\left(8-\left(\alpha+p_{1}\right)^{2}\right) c_{2}+4\left(2-\alpha p_{1}\right)\left(6+2 p_{1}-\left(\alpha+p_{1}\right)^{2}\right)}{48+\alpha p_{1}\left(\alpha+p_{1}\right)^{2}-4\left(\alpha^{2}+6 \alpha p_{1}-p_{1}^{2}\right)}
$$

The trace $o$ and determent of Jacobian matrix (22) is

$$
\begin{aligned}
\operatorname{Tr} & =2-\lambda_{1} p_{b 1}^{c} \frac{8-\left(\alpha+p_{1}\right)^{2}}{4\left(2-\alpha p_{1}\right)}-\lambda_{2} p_{2}^{c} \frac{8-2 \alpha p_{1}}{4\left(2-\alpha p_{1}\right)}, \\
\operatorname{Det} & =\left(1-\lambda_{1} p_{b 1}^{c} \frac{8-\left(\alpha+p_{1}\right)^{2}}{4\left(2-\alpha p_{1}\right)}\right)\left(1-\lambda_{2} p_{2}^{c} \frac{8-2 \alpha p_{1}}{4\left(2-\alpha p_{1}\right)}\right)-\lambda_{1} \lambda_{2} p_{b 1}^{c} p_{2}^{c} \frac{\left(4-\alpha p_{1}-p_{1}^{2}\right)\left(4-2 \alpha p_{1}\right)}{16\left(2-\alpha p_{1}\right)^{2}} .
\end{aligned}
$$

The characteristic equation is $x^{2}-\operatorname{Tr} x+$ Det $=0$. 
For $\operatorname{Tr}^{2}-4 \operatorname{Det}=\left(\lambda_{1} p_{b 1}^{c}\left(\left(8-\left(\alpha+p_{1}\right)^{2}\right) / 4\left(2-\alpha p_{1}\right)\right)-\right.$ $\left.\lambda_{2} p_{2}^{c}\left(\left(8-2 \alpha p_{1}\right) / 4\left(2-\alpha p_{1}\right)\right)\right)^{2}+4 \lambda_{1} \lambda_{2} p_{b 1}^{c} p_{2}^{c}\left(\left(4-\alpha p_{1}-\right.\right.$ $\left.\left.p_{1}^{2}\right)\left(4-2 \alpha p_{1}\right) / 16\left(2-\alpha p_{1}\right)^{2}\right)>0$, so the equation roots are real.

According to the Jury condition [30], the system stable condition is

$$
\left\{\begin{array}{l}
\text { (a) } 1-\operatorname{Det}>0, \\
\text { (b) } 1-\operatorname{Tr}+\operatorname{Det}>0 \\
\text { (c) } 1+\operatorname{Tr}+\operatorname{Det}>0 .
\end{array}\right.
$$

In equation (25), if condition (a) is not satisfied, there is a complex conjugate pair of eigenvalues lying outside the unit circle. If condition (b) is not satisfied, there is a root is less than -1 . If condition (c) is not satisfied, there is a root is larger than 1 [28].

Condition (a) in equation (16) is

$$
\begin{aligned}
1-\text { Det }= & 1-\left(1-\lambda_{1} p_{b 1}^{c} \frac{8-\left(\alpha+p_{1}\right)^{2}}{4\left(2-\alpha p_{1}\right)}\right)\left(1-\lambda_{2} p_{2}^{c} \frac{8-2 \alpha p_{1}}{4\left(2-\alpha p_{1}\right)}\right) \\
& +\lambda_{1} \lambda_{2} p_{b 1}^{c} p_{2}^{c} \frac{\left(4-\alpha p_{1}-p_{1}^{2}\right)\left(4-2 \alpha p_{1}\right)}{16\left(2-\alpha p_{1}\right)^{2}} \\
= & \lambda_{1} p_{b 1}^{c} \frac{8-\left(\alpha+p_{1}\right)^{2}}{4\left(2-\alpha p_{1}\right)}+\lambda_{2} p_{2}^{c} \frac{8-2 \alpha p_{1}}{4\left(2-\alpha p_{1}\right)} \\
& +\lambda_{1} \lambda_{2} p_{b 1}^{c} p_{2}^{c} \frac{\left(4-\alpha p_{1}-p_{1}^{2}\right)\left(4-2 \alpha p_{1}\right)-\left(8-\left(\alpha+p_{1}\right)^{2}\right)\left(8-2 \alpha p_{1}\right)}{16(2-\alpha p)^{2}}>0 .
\end{aligned}
$$

So, the adjustment speed $\lambda_{1}$ satisfied

$$
0<\lambda_{1}<\frac{-4\left(2-\alpha p_{1}\right) \lambda_{2} p_{2}^{c}\left(8-2 \alpha p_{1}\right)}{4\left(2-\alpha p_{1}\right) p_{b 1}^{c}\left(8-\left(\alpha+p_{1}\right)^{2}\right)+\lambda_{2} p_{b 1}^{c} p_{2}^{c}\left(\left(4-\alpha p_{1}-p_{1}^{2}\right)\left(4-2 \alpha p_{1}\right)-\left(8-\left(\alpha+p_{1}\right)^{2}\right)\left(8-2 \alpha p_{1}\right)\right)}
$$

Condition (b) in equation (16) is

$$
\begin{aligned}
1-\operatorname{Tr}+\operatorname{Det}= & 1-\left(2-\lambda_{1} p_{b 1}^{c} \frac{8-\left(\alpha+p_{1}\right)^{2}}{4\left(2-\alpha p_{1}\right)}-\lambda_{2} p_{2}^{c} \frac{8-2 \alpha p_{1}}{4\left(2-\alpha p_{1}\right)}\right) \\
& +\left(1-\lambda_{1} p_{b 1}^{c} \frac{8-\left(\alpha+p_{1}\right)^{2}}{4\left(2-\alpha p_{1}\right)}\right)\left(1-\lambda_{2} p_{2}^{c} \frac{8-2 \alpha p_{1}}{4\left(2-\alpha p_{1}\right)}\right)-\lambda_{1} \lambda_{2} p_{b 1}^{c} p_{2}^{c} \frac{\left(4-\alpha p_{1}-p_{1}^{2}\right)\left(4-2 \alpha p_{1}\right)}{16\left(2-\alpha p_{1}\right)^{2}} \\
= & \lambda_{1} \lambda_{2} p_{b 1}^{c} p_{2}^{c}\left(\frac{\left(8-\left(\alpha+p_{1}\right)^{2}\right)\left(8-2 \alpha p_{1}\right)-\left(4-\alpha p_{1}-p_{1}^{2}\right)\left(4-2 \alpha p_{1}\right)}{16\left(2-\alpha p_{1}\right)^{2}}\right)>0,
\end{aligned}
$$

so this condition is easily satisfied.

Condition (c) in equation (16) is 


$$
\begin{aligned}
1+\operatorname{Tr}+\operatorname{Det}= & 1+\left(2-\lambda_{1} p_{b 1}^{c} \frac{8-\left(\alpha+p_{1}\right)^{2}}{4\left(2-\alpha p_{1}\right)}-\lambda_{2} p_{2}^{c} \frac{8-2 \alpha p_{1}}{4\left(2-\alpha p_{1}\right)}\right)+\left(1-\lambda_{1} p_{b 1}^{c} \frac{8-\left(\alpha+p_{1}\right)^{2}}{4\left(2-\alpha p_{1}\right)}\right)\left(1-\lambda_{2} p_{2}^{c} \frac{8-2 \alpha p_{1}}{4\left(2-\alpha p_{1}\right)}\right) \\
& -\lambda_{1} \lambda_{2} p_{b 1}^{c} p_{2}^{c} \frac{\left(4-\alpha p_{1}-p_{1}^{2}\right)\left(4-2 \alpha p_{1}\right)}{16\left(2-\alpha p_{1}\right)^{2}} \\
= & 4-2 \lambda_{1} p_{b 1}^{c} \frac{8-\left(\alpha+p_{1}\right)^{2}}{4\left(2-\alpha p_{1}\right)}-2 \lambda_{2} p_{2}^{c} \frac{8-2 \alpha p_{1}}{4\left(2-\alpha p_{1}\right)} \\
& +\lambda_{1} \lambda_{2} p_{b 1}^{c} p_{2}^{c}\left(\frac{\left(8-\left(\alpha+p_{1}\right)^{2}\right)\left(8-2 \alpha p_{1}\right)-\left(4-\alpha p_{1}-p_{1}^{2}\right)\left(4-2 \alpha p_{1}\right)}{16\left(2-\alpha p_{1}\right)^{2}}\right)>0 .
\end{aligned}
$$
satisfy

So, the adjustment speed of sharing platform $\lambda_{1}$ should

$$
0<\lambda_{1}<\frac{8\left(8\left(2-\alpha p_{1}\right)-2 \lambda_{2} p_{2}^{c}\left(8-2 \alpha p_{1}\right)\right)\left(2-\alpha p_{1}\right)}{8\left(2-\alpha p_{1}\right) p_{b 1}^{c}\left(8-\left(\alpha+p_{1}\right)^{2}\right)-\lambda_{2} p_{b 1}^{c} p_{2}^{c}\left(\left(8-\left(\alpha+p_{1}\right)^{2}\right)\left(8-2 \alpha p_{1}\right)-\left(4-\alpha p_{1}-p_{1}^{2}\right)\left(4-2 \alpha p_{1}\right)\right)}
$$

We illustrate the stable area in Figure 2. The blue area I is the stable area, and this area satisfied all conditions; area II is the area that satisfied condition (a) and condition (b), area III is the area that satisfied condition (b). Figure 1 shows that when the adjustment speed $\lambda_{1}$ and $\lambda_{2}$ increases, system (14) becomes flip bifurcation and then becomes a chaotic state.

\section{Simulation}

In this section, numerical examples are presented to illustrate the dynamic behavior of the sharing platform competition model. We present some numerical evidence for the chaotic of the sharing platform system. In the following, we illustrate the three factors' effects on stability of system (14). Three factors are adjustment speed of sharing platform's, exogenous price, and traditional firm cost.

4.1. Adjustment Speed of Sharing Platform. We first fixed the parameter set $\alpha=0.1, p_{1}=0.7, c_{2}=0.5$, and $\lambda_{2}=0.9$; we then illustrate the $\lambda_{1}$ effect on system stability.

Figure 3 describes the bifurcation diagram with respect to $\lambda_{1}$. As shown in Figure $3, p_{b 1}, p_{s 1}, p_{2}$ are stable when $\lambda_{1}$ is small; as $\lambda_{1}$ increases, system (14) becomes a bifurcation, and finally, the system goes into a chaotic state. We can see that when the system is stable, $p_{2}$ is larger than $p_{b 1}$, while $p_{s 1}$ is smaller than zero. Sharing platform's fee decision shows a standard two-sided characteristic.

Figure 4 shows largest Lyapunov exponent (LE) with respect to $\lambda_{1}$ corresponding to Figure 3 ; LE is used to market the system chaos. When $0<\lambda_{1}<1.648$, the LE is negative; system (14) is in a stable state. When $\lambda_{1} \approx 1.648$, the LE equals to zero, which means that system (14) goes into bifurcation. When $\lambda_{1}$ is larger than 2.766, LE is mostly positive; this means system (14) loses control and enters a chaotic state. Figure 5 shows strange attractor of system (14) when $\lambda_{1}=2.522$. We can see that although system (14) is in a chaotic state, $p_{b 1}$ and $p_{2}$ are still in a certain scale.

4.2. Sharing Platform Price. In this section, we show sharing platform price effects on system (14) stability. We first fixed the parameter set $\alpha=0.1, c_{2}=0.5, \lambda_{1}=2.6$, and $\lambda_{2}=0.9$.

Figure 6 shows stable area of $\left(\lambda_{1}, \lambda_{2}\right)$ with different values of $p_{1}$. It can be seen that the system stable area changes with $p_{1}$; when $p_{1}$ is small in a certain value, system (14) would be stable. When $p_{1}$ increases, the stable area boundary in $\lambda_{1}$ would be larger, while the stable area boundary in $\lambda_{2}$ would be smaller; this means that when $p_{1}$ increases, system would be more stable in adjustment speed of the sharing platform, while more vulnerable in adjustment speed of traditional firm.

Figure 7 describes the bifurcation diagram with respect to $p_{1}$. As shown in Figure $7, p_{b 1}, p_{s 1}, p_{2}$ are in a chaotic state when $p_{1}$ is small; as $p_{1}$ increases, system (14) goes into 2period bifurcation, and finally, the system goes into a stable state. We can see that when the system is stable, $p_{2}$ is larger than $p_{b 1}$. As $p_{1}$ increases, $p_{2}$ is larger and $p_{b 1}$ and $p_{s 1}$ are smaller. That means higher $p_{1}$ is a benefit for system stability, but the sharing platform gets lost in benefit.

Figure 8 shows the largest Lyapunov exponent with respect to $p_{1}$ corresponding to Figure 7 . When $p_{1}<0.455$, the LE is mostly positive; system (14) is in a chaotic state. When $\lambda_{1} \approx 0.445$, the LE equals to zero, which means that system (14) changes from the chaotic state into 2-period bifurcation. When $p_{1}$ is larger than 0.934 , LE is negative; system (14) is in a stable state. Figure 9 shows the strange attractor of system (14) when $p_{1}=0.300$. We can see that $p_{b 1}$ and $p_{2}$ are still in a certain scale. 


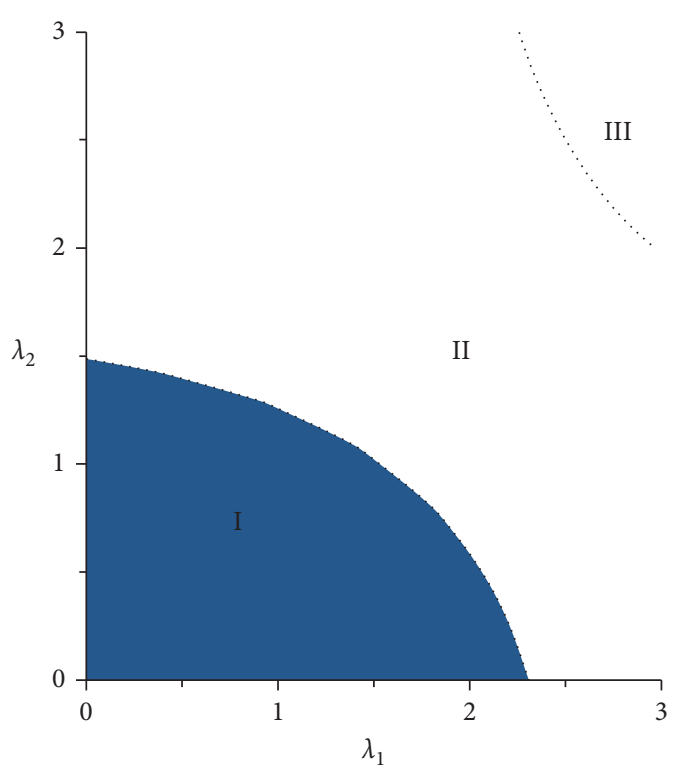

FIgURE 2: Stable area of system (14).

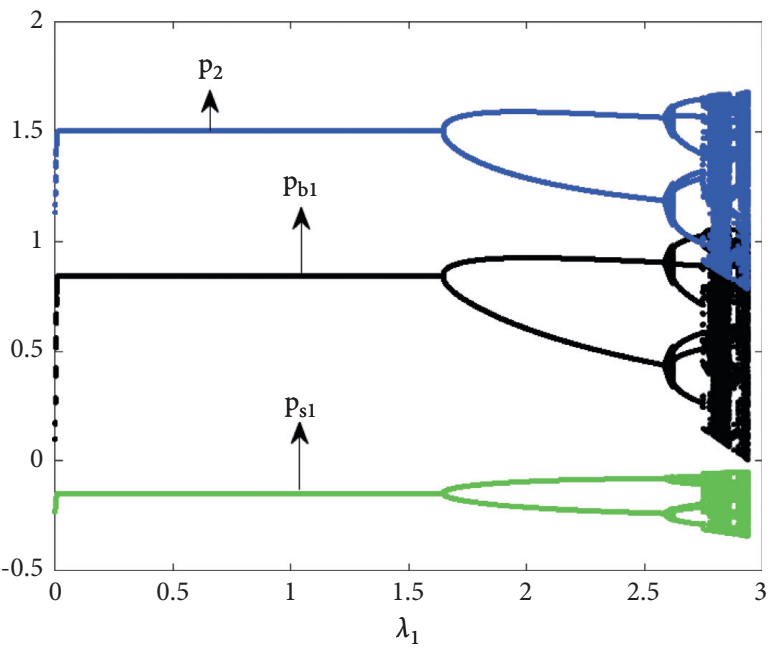

Figure 3: Bifurcation diagram with respect to $\lambda_{1}$ when $\alpha=0.1, p_{1}=0.7, c_{2}=0.5$, and $\lambda_{2}=0.9$.

4.3. Cost Effects. In this section, we illustrate traditional cost effects on system (14) stability. We first fixed the parameter set, $\alpha=0.1, \lambda_{1}=2.6, \lambda_{2}=1.2$, and $p_{1}=0.7$.

Figure 10 shows stable area of $\left(\lambda_{1}, \lambda_{2}\right)$ with different values of $c_{2}$. It can be seen that the system stable area changes with $c_{2}$; when $c_{2}$ is small in a certain value, the system (14) would be stable. When $c_{2}$ increases, the stable area boundary would be smaller. This means that when $c_{2}$ is lower, the system would be more stable.

Figure 11 describes the bifurcation diagram with respect to $c_{2}$. As shown in Figure $11, p_{b 1}, p_{s 1}, p_{2}$ are stable when $c_{2}$ is small; as $c_{2}$ increases, system (14) goes into 2-period bifurcation, and finally, the system goes into a chaotic state. We can see that when the system is stable, with $c_{2}$ increasing, $p_{2}$ increases quicker than $p_{b 1}$, while $p_{s 1}$ decreases. That means traditional firm is more sensitive to $c_{2}$.

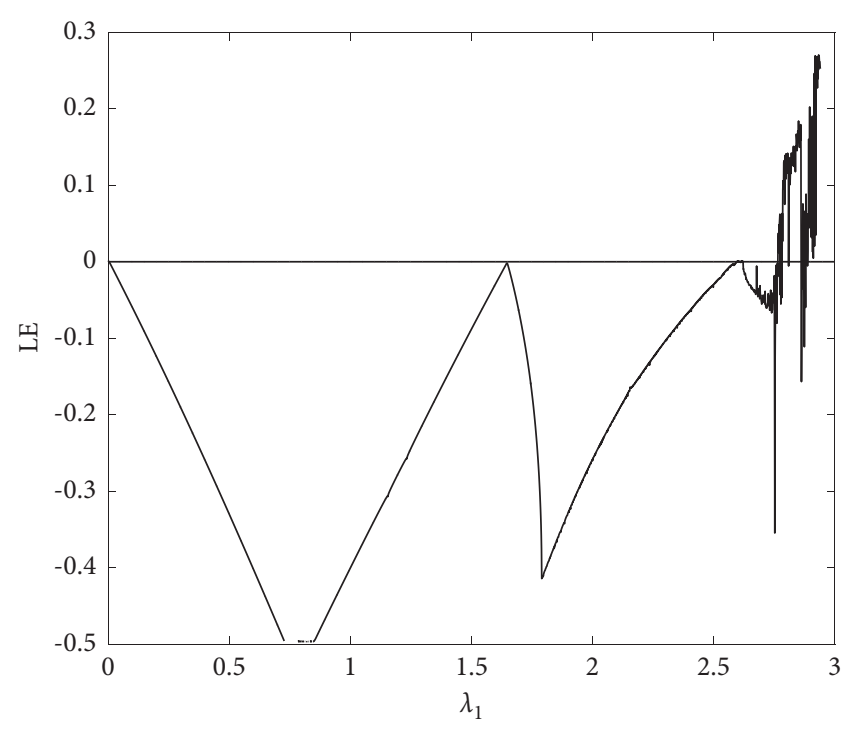

FIgURE 4: The largest Lyapunov exponent with respect to $\lambda_{1}$ corresponding to Figure 3.

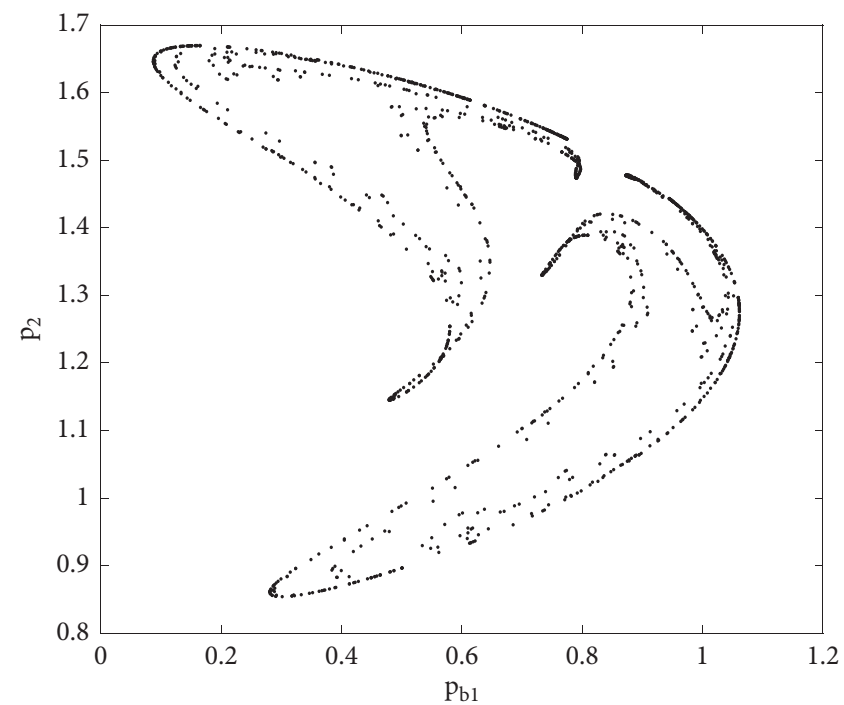

FIGURE 5: Strange attractor of system (14) when $\lambda_{1}=2.522$ and $\alpha=0.1, p_{1}=0.7, c_{2}=0.5, \lambda_{1}=2.831$, and $\lambda_{2}=0.9$.

Figure 12 shows the largest Lyapunov exponent with respect to $c_{2}$ corresponding to Figure 11 . When $0<c_{2}<1.367$, the LE is negative; system (14) is in a stable state. When $c_{2} \approx 1.367$, the LE equals to zero, which means that system (14) changes from stable state into 2-period bifurcation. When $c_{2}$ is larger than 1.781, LE is mostly positive; the system (14) is in a chaotic state. Figure 13 shows the strange attractor of system (14) when $c_{2}=1.981$. We can see that $p_{b 1}$ and $p_{2}$ are still in a certain scale.

4.4. Delay Feedback Control. We can see that when system (14) is in a chaotic state, the system is of great sensitivity under initial conditions. When the system becomes chaotic, it means that the sharing market becomes out of control; 


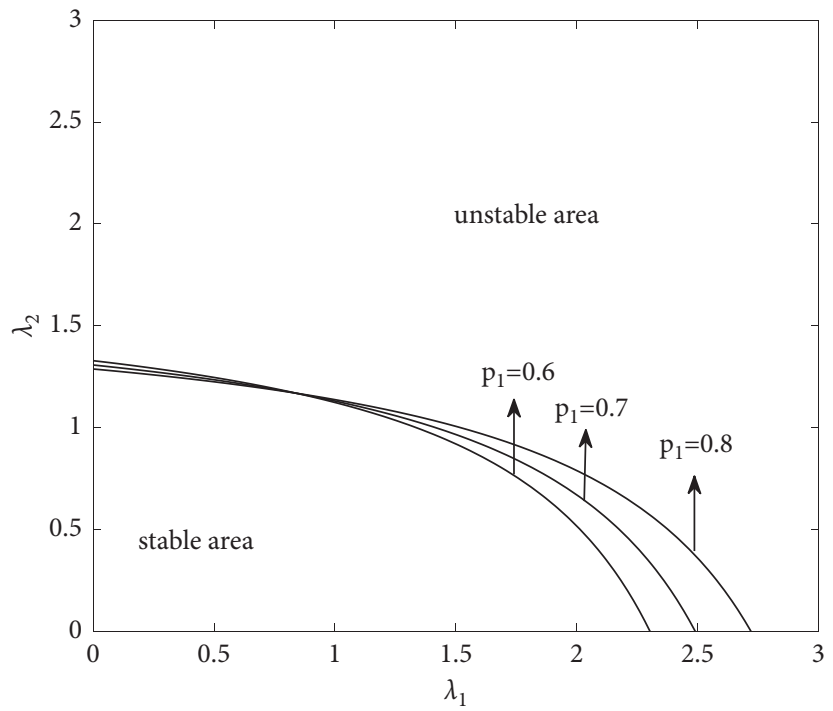

Figure 6: Stable area of $\left(\lambda_{1}, \lambda_{2}\right)$ with different values of $p_{1}$.

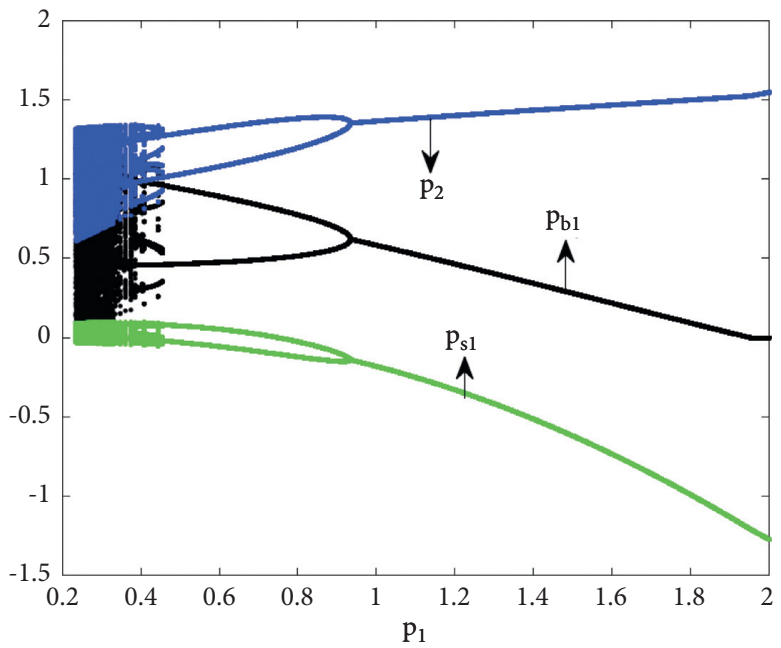

Figure 7: Bifurcation diagram with respect to $p_{1}$ when $\alpha=0.1, c_{2}=0.5, \lambda_{1}=2.6$, and $\lambda_{2}=0.9$.

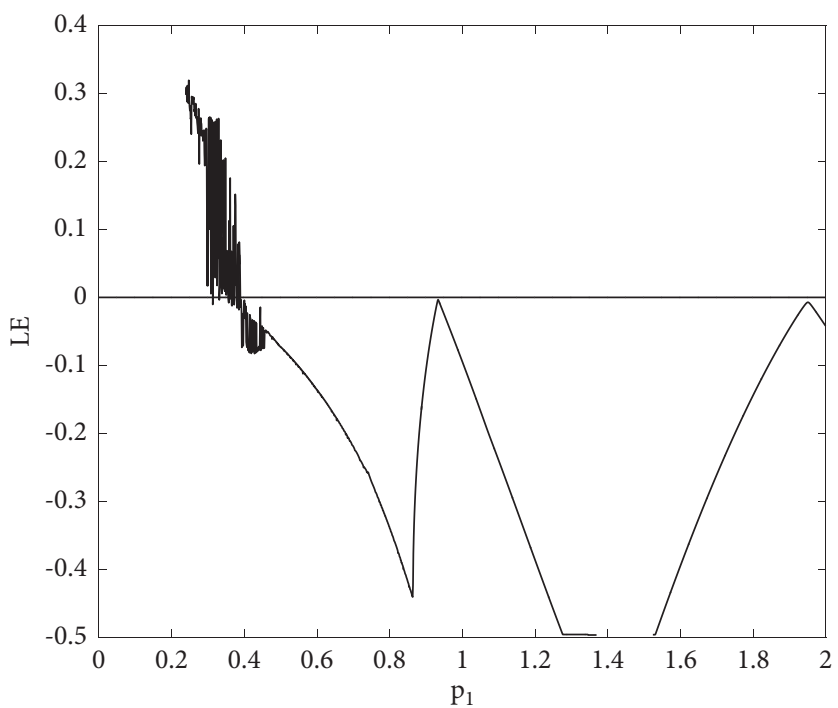

Figure 8: The largest Lyapunov exponent with respect to $p_{1}$ corresponding to Figure 7. 


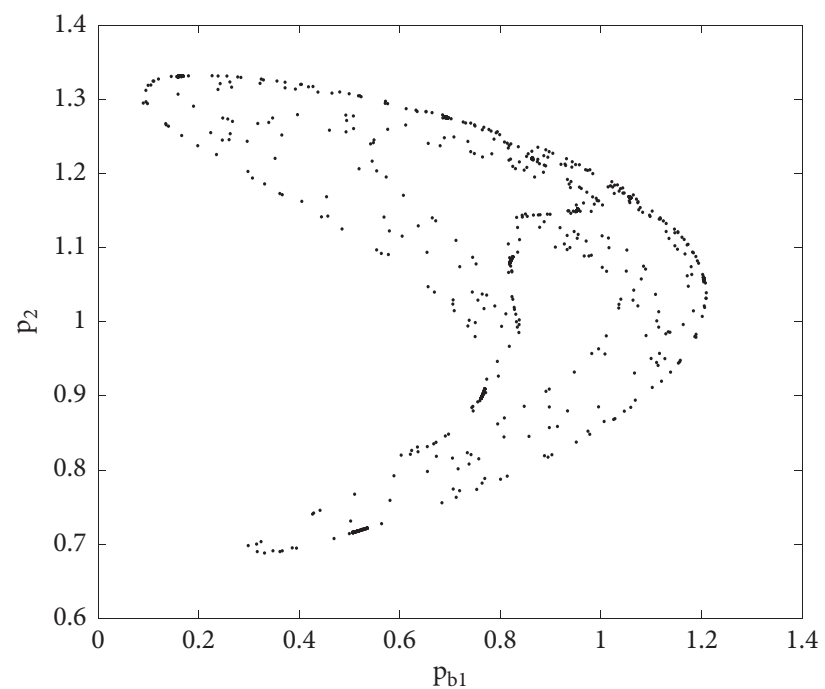

FIGURE 9: Strange attractor of system (14) when $p_{1}=0.300$.

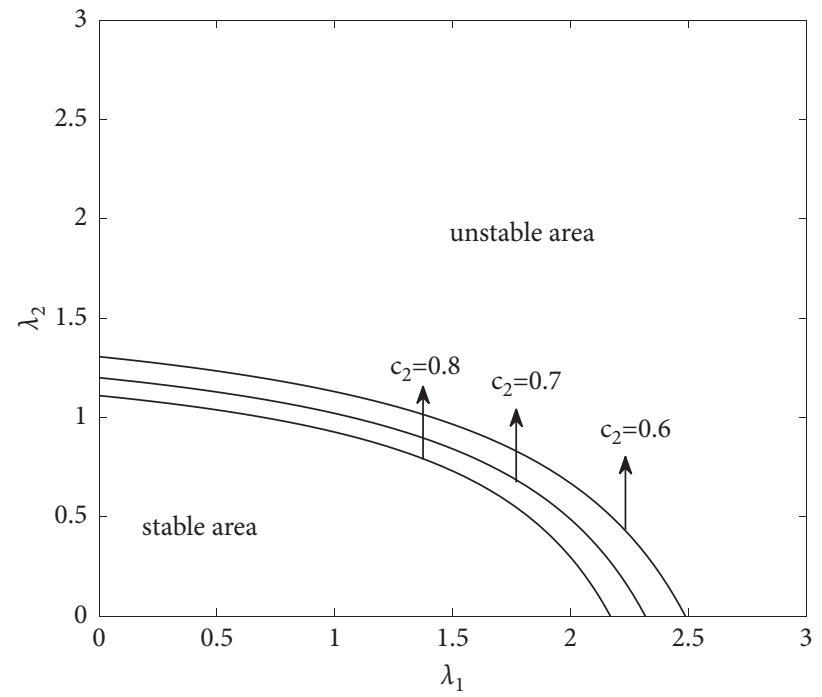

Figure 10: Stable area of $\left(\lambda_{1}, \lambda_{2}\right)$ with different values of $c_{2}$.

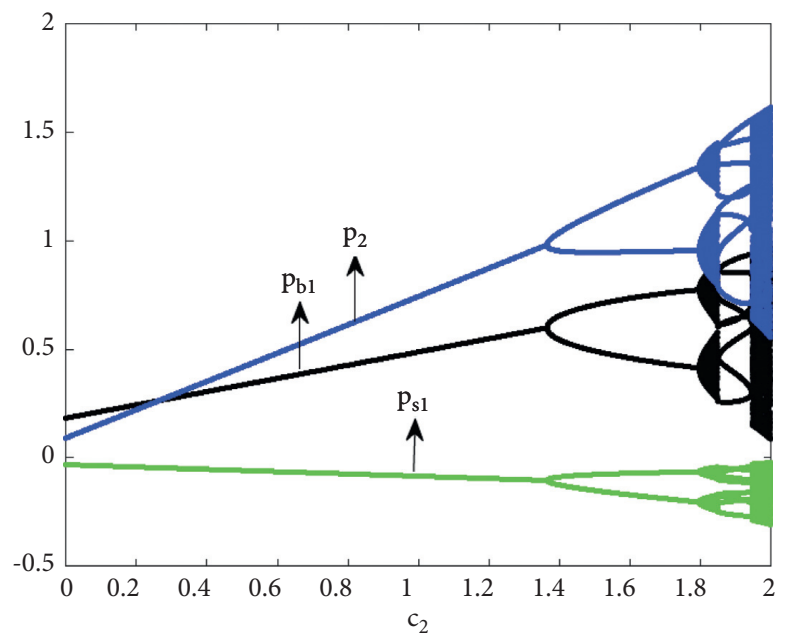

Figure 11: Bifurcation diagram with respect to $c_{2}$ when $\alpha=0.1, p_{1}=0.7, \lambda_{1}=2.6$, and $\lambda_{2}=1.2$. 


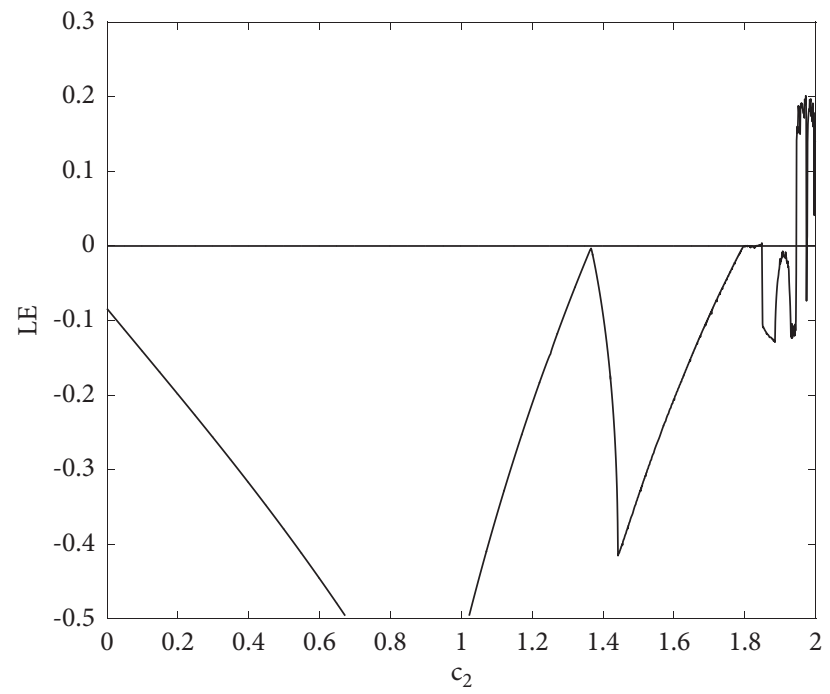

FIgURE 12: The largest Lyapunov exponent with respect to $c_{2}$ corresponding to Figure 11.

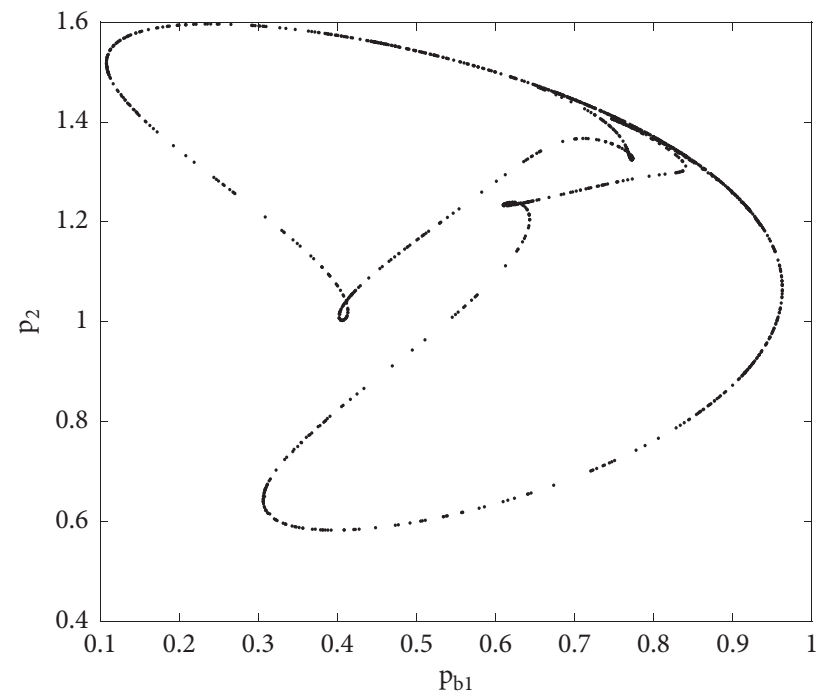

FIGURE 13: Strange attractor of system (14) when $c_{2}=1.981$ and $\alpha=0.1, p_{1}=0.7, \lambda_{1}=2.6$, and $\lambda_{2}=1.2$.

feedback control becomes a general way to control the chaos state [31].

We use the control mechanism: $F\left(p_{b 1}(t+1)\right)=k\left(p_{b 1}(t)-p_{b 1}(t+1)\right)$, where $k$ is the feedback control parameter. The sharing platform adjusts its consumer fee to alleviate the chaotic state. Then, the system becomes

$$
\left\{\begin{array}{l}
p_{b 1}(t+1)=p_{b 1}(t)+\lambda_{1} p_{b 1}(t) \frac{\partial \pi_{1}}{\partial p_{b 1}}+F\left(p_{b 1}(t+1)\right) \\
p_{2}(t+1)=p_{2}(t)+\lambda_{2} p_{2}(t) \frac{\partial \pi_{2}}{\partial p_{2}}
\end{array}\right.
$$

Then, the feedback control system can be written as

$$
\left\{\begin{array}{l}
p_{b 1}(t+1)=p_{b 1}(t)+\frac{\lambda_{1} p_{b 1}(t)}{1+k} \frac{\partial \pi_{1}}{\partial p_{b 1}} \\
p_{2}(t+1)=p_{2}(t)+\lambda_{2} p_{2}(t) \frac{\partial \pi_{2}}{\partial p_{2}}
\end{array}\right.
$$

For controlling the chaotic sharing economy systems, we use the time-delayed feedback control [26]. We set parameter: $\alpha=0.1, p_{1}=0.7, c_{2}=0.5$, and $\lambda_{2}=0.9$ when $\lambda_{1}=2.831$; as former simulation in Figure 3, we know that the system is in a chaotic state.

We simulate feedback control when $k=0.5$ and $k=0.8$. Figure 14 shows the original system is in the chaos state after several periods iteration, while under the delay feedback control $k=0.5$, the system becomes double bifurcation and the chaos state alleviates; while $k=0.8$, the system becomes stable. 

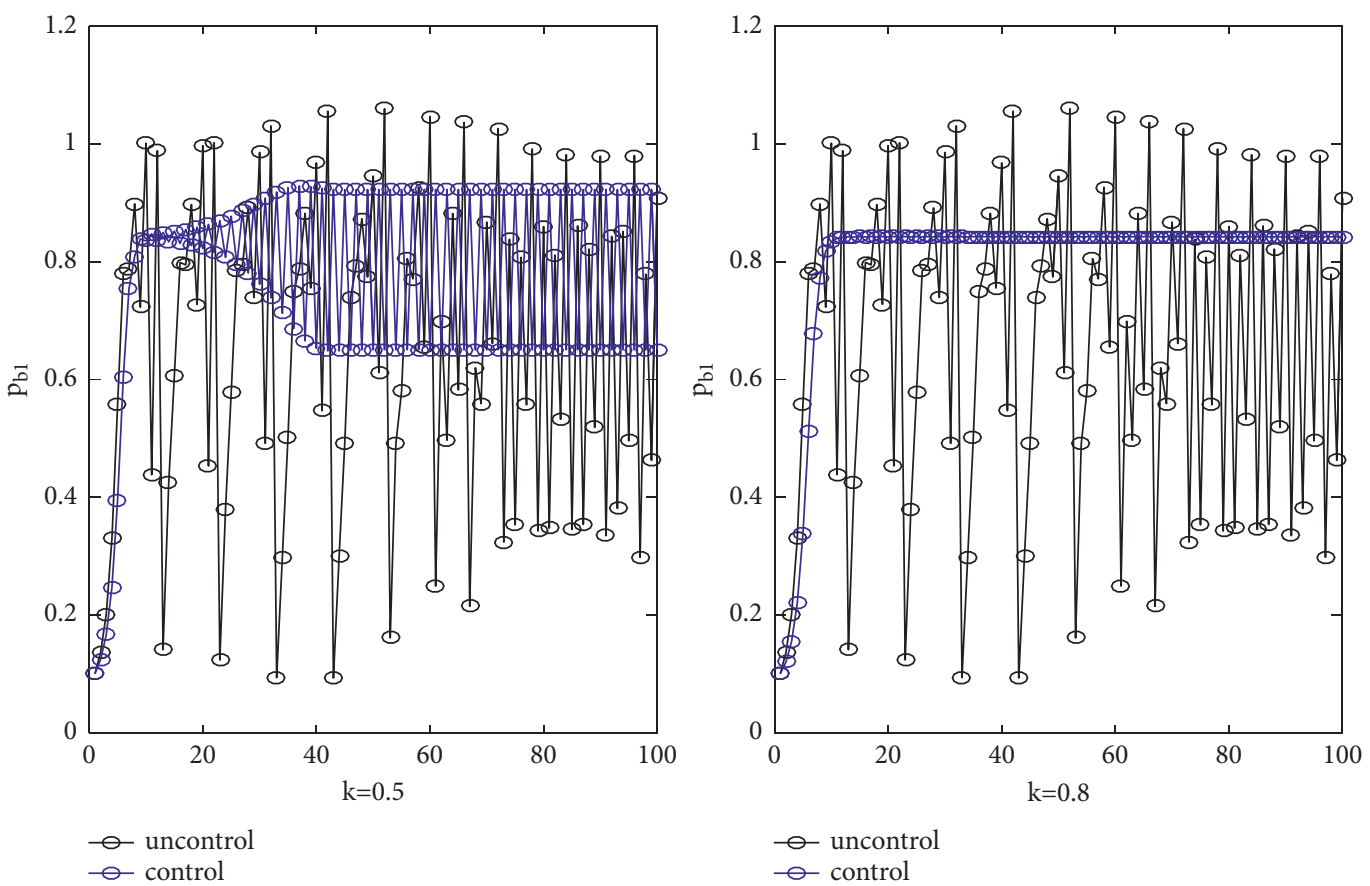

FIGURE 14: Delay feedback control when $k=0.5,0.8$.

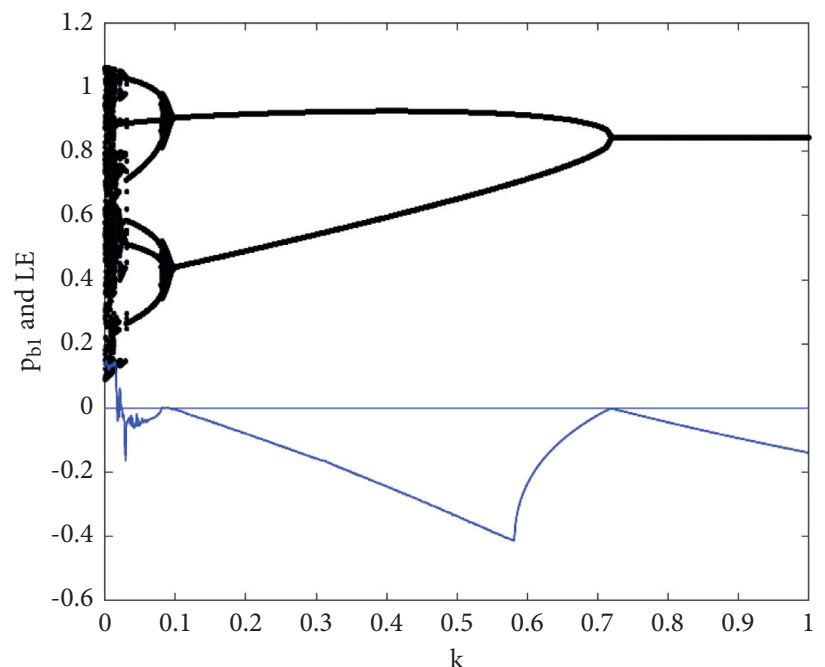

Figure 15: Bifurcation diagram and the largest Lyapunov exponent with respect to $k$ when $\alpha=0.1, p_{1}=0.7, c_{2}=0.5$, $\lambda_{1}=2.831$, and $\lambda_{2}=0.9$.

Figure 15 shows bifurcation diagram and the largest Lyapunov exponent with respect to $k$. Figure 15 shows that when the adjustment speed becomes higher, the chaos state alleviates to double bifurcation in $k \approx 0.093$ and becomes a stable state in $k \approx 0.721$. This indicates that system (14) can get rid of chaos successfully when the controlling parameter becomes larger.

\section{Conclusion}

Sharing economy has become a major trend in recent years. Sharing platform reduces transaction costs and enhances consumer welfare. The competition between sharing economy and traditional firm becomes a major concern of many researchers. Our paper focuses on two factors: the first is sharing platform's two-sided market structure; the second is, in sharing platform competition, sharing platform and traditional firm are both bounded rational.

We build a dynamic sharing platform competition model and then present the fixed points and the stability conditions of the bifurcation of the dynamic model. At last, we simulate the adjustment speed of sharing platform, sharing platform price, and costs of traditional firm effects on system stability. With the aid of stable area, bifurcation 
diagram, the largest Lyapunov exponent, and strange attractor, we present the following main conclusions:

(1) Adjustment speed of sharing platform has a positive effect on system stability. When adjustment speed of sharing platform increases, the system is more likely to be in a chaotic state.

(2) Price of the sharing platform has a bilateral effect on system stability. When it increases, in sharing platform side, the system is more stable while the traditional firm is more vulnerable. Sharing platform changes with traditional firm price in the reverse direction.

(3) Traditional firm cost has a positive effect on system stability. Traditional firm cost has positive effects on traditional price but has a bilateral effect on sharing platform fees.

(4) Feedback control can alleviate the chaotic state of the system. By adjusting the feedback control parameter $k$, the sharing platform and traditional firm can make the system stable.

\section{Data Availability}

The data used to support the findings of this study are available upon request to the author.

\section{Conflicts of Interest}

The authors declare that they have no conflicts of interest.

\section{Acknowledgments}

This research was supported by the Natural Science Foundation Project of Guizhou Provincial Education Department (Qian Jiao He KY zi [2019] 207)

\section{References}

[1] G. M. Eckhardt, M. B. Houston, B. Jiang, C. Lamberton, A. Rindfleisch, and G. Zervas, "Marketing in the sharing economy," Journal of Marketing, vol. 83, no. 5, pp. 5-27, 2019.

[2] R. Calo and A. Rosenblat, "The taking economy: uber, information, and power," Columbia Law Review, vol. 117, pp. 1623-1690, 2017.

[3] A. Goldfarb and C. Tucker, "Digital economics," Journal of Economic Literature, vol. 57, no. 1, pp. 3-43, 2019.

[4] Y. Nie, "How can the taxi industry survive the tide of ridesourcing? evidence from Shenzhen, China," Transportation Research Part C: Emerging Technologies, vol. 79, pp. 242-256, 2017.

[5] J. J. Yu, C. S. Tang, Z. M. Shen, and X. M. Chen, "A balancing act of regulating on-demand ride services," Management Science, vol. 66, pp. 2975-2992, 2019.

[6] F. Bardhi and G. M. Eckhardt, "Access-based consumption: the case of car sharing," Journal of Consumer Research, vol. 39, no. 4, pp. 881-898, 2012.

[7] A. Hagiu and H. Hałaburda, "Information and two-sided platform profits," International Journal of Industrial Organization, vol. 34, pp. 25-35, 2014.
[8] J. Pei, P. Yan, S. Kumar, and X. Liu, "How to react to internal and external sharing in $\mathrm{B} 2 \mathrm{C}$ and $\mathrm{C} 2 \mathrm{C}$," Production and Operations Management, vol. 30, no. 1, pp. 145-170, 2021.

[9] L. Tian and B. Jiang, "Effects of consumer-to-consumer product sharing on distribution channel," Production and Operations Management, vol. 27, no. 2, pp. 350-367, 2018.

[10] G. P. Cachon, K. M. Daniels, and R. Lobel, "The role of surge pricing on a service platform with self-scheduling capacity," Manufacturing \& Service Operations Management, vol. 19, no. 3, pp. 368-384, 2017.

[11] R. Seamans and F. Zhu, "Responses to entry in multi-sided markets: the impact of craigslist on local newspapers," Management Science, vol. 60, no. 2, pp. 476-493, 2014.

[12] J.-C. Rochet and J. Tirole, "Platform competition in two-sided markets," Journal of the European Economic Association, vol. 1, no. 4, pp. 990-1029, 2003.

[13] M. Armstrong, "Competition in two-sided markets," The RAND Journal of Economics, vol. 37, no. 3, pp. 668-691, 2006.

[14] D. Bardey, H. Cremer, and J.-M. Lozachmeur, "Competition in two-sided markets with common network externalities," Review of Industrial Organization, vol. 44, no. 4, pp. 327-345, 2014.

[15] P. Belleflamme and E. Toulemonde, "Negative intra-group externalities in two-sided markets," International Economic Review, vol. 50, no. 1, pp. 245-272, 2009.

[16] A. Ambrus and R. Argenziano, "Asymmetric networks in twosided markets," American Economic Journal: Microeconomics, vol. 1, no. 1, pp. 17-52, 2009.

[17] B. Harris, "Uber, lyft, and regulating the sharing economy," Seattle University Law Review, vol. 41, pp. 269-285, 2017.

[18] M. Hu, "From the classics to new tunes: a neoclassical view on sharing economy and innovative marketplaces," Production and Operations Management, vol. 30, no. 6, pp. 1668-1685, 2020.

[19] K. Rong, F. Xiao, and Y. Wang, "Redundancy in the sharing economy," Resources, Conservation and Recycling, vol. 151, Article ID 104455, 2019.

[20] F. He and Z.-J. M. Shen, "Modeling taxi services with smartphone-based e-hailing applications," Transportation Research Part C: Emerging Technologies, vol. 58, pp. 93-106, 2015.

[21] F. Ye, D. Ni, and K. W. Li, "Competition between manufacturers and sharing economy platforms: an owner base and sharing utility perspective," International Journal of Production Economics, vol. 234, Article ID 108022, 2021.

[22] J. Andaluz, A. A. Elsadany, and G. Jarne, "Nonlinear cournot and bertrand-type dynamic triopoly with differentiated products and heterogeneous expectations," Mathematics and Computers in Simulation, vol. 132, pp. 86-99, 2017.

[23] J.-G. Du, Y.-Q. Fan, Z.-H. Sheng, and Y.-Z. Hou, "Dynamics analysis and chaos control of a duopoly game with heterogeneous players and output limiter," Economic Modelling, vol. 33, pp. 507-516, 2013.

[24] L. Fanti and L. Gori, "The dynamics of a differentiated duopoly with quantity competition," Economic Modelling, vol. 29, no. 2, pp. 421-427, 2012.

[25] T. Dubiel-Teleszynski, "Nonlinear dynamics in a heterogeneous duopoly game with adjusting players and diseconomies of scale," Communications in Nonlinear Science and Numerical Simulation, vol. 16, no. 1, pp. 296-308, 2011.

[26] K. Pyragas, "Delayed feedback control of chaos," Philosophical Transactions of the Royal Society A: Mathematical, Physical and Engineering Sciences, vol. 364, no. 1846, pp. 2309-2334, 2006. 
[27] H. Zou and D. He, "Technology sharing game from ecological perspective," Applied Mathematics and Nonlinear Sciences, vol. 6, pp. 81-92, 2021.

[28] J. P. Choi, B. Jullien, and Y. Lefouili, "Tying in two-sided markets with multi-homing: corrigendum and comment," Journal of Industrial Economics, vol. 65, no. 4, 2017.

[29] A. A. Elsadany, "Dynamics of a cournot duopoly game with bounded rationality based on relative profit maximization," Applied Mathematics and Computation, vol. 294, pp. 253-263, 2017.

[30] Y. Peng, Q. Lu, and Y. Xiao, “A dynamic stackelberg duopoly model with different strategies," Chaos, Solitons \& Fractals, vol. 85, pp. 128-134, 2016.

[31] K. Pyragas, "Continuous control of chaos by self-controlling feedback," Physics Letters A, vol. 170, no. 6, pp. 421-428, 1992. 